\title{
Ann Arbor Stage I Nodal Marginal Zone Lymphoma
}

National Cancer Institute

\section{Source}

National Cancer Institute. Ann Arbor Stage I Nodal Marginal Zone Lymphoma. NCI Thesaurus. Code C8864.

Ann Arbor Classification: Stage I: Involvement of a single lymph node region (I); or localized involvement of a single extralymphatic organ or site in the absence of any lymph node involvement (IE).-2003 